\title{
Induction of RhoA gene expression by interleukin-4 in cultured human bronchial smooth muscle cells
}

\author{
Yoshihiko ChiBA ${ }^{1}$, Kumiko GoTO ${ }^{1}$, Makiko Momata ${ }^{1}$, \\ Taiki KoBAYASHI ${ }^{1}$ and Miwa MisAwA ${ }^{1}$ \\ ${ }^{1}$ Department of Pharmacology, School of Pharmacy, Hoshi University, Tokyo, Japan
}

Received May 12, 2010; Accepted May 26, 2010

\begin{abstract}
RhoA, a small GTPase, is one of the key proteins of smooth muscle contraction. In allergic asthma, an upregulation of RhoA in bronchial smooth muscle has been suggested. However, the mechanism of its upregulation has not yet been clarified. In the present study, the effects of interleukin-4 (IL-4), one of the T-helper 2 cytokines, on RhoA mRNA expression and promoter activity of RhoA gene were examined in cultured human bronchial smooth muscle cells (hBSMCs). The quantitative real-time RT-PCR analyses revealed that incubation of hBSMCs with IL-4 (10, 30 and $100 \mathrm{ng} / \mathrm{mL}$, for $24 \mathrm{hr}$ ) caused an increase in RhoA mRNA in a concentration-dependent manner. In luciferase reporter gene assay using hBSMCs that were transfected with luciferase constructs and were then stimulated with IL$4(100 \mathrm{ng} / \mathrm{mL})$, an importance of the most proximal STAT6 binding region (78-70 bp upstream of the transcription initiation site) was suggested. It is thus possible that IL-4 is capable of upregulating RhoA by promoting its transcription in hBSMCs. The proximal STAT6 binding region is required for the IL-4-induced increase in promoter activity of the human RhoA gene.
\end{abstract}

Key words: RhoA, interleukin-4, allergic bronchial asthma, bronchial smooth muscle hyperresponsiveness

\section{Introduction}

The dramatic increase in the number of asthma cases over the last decades is of great concern for public health in the world (Eder et al., 2006). Increased airway narrowing in response to nonspecific stimuli is a characteristic feature of human obstructive diseases, including bronchial asthma. The abnormality is an important sign of the disease, although the pathophysiological variations leading to the hyperresponsiveness are unclear now. It has been suggested that one of the factors that contribute to the exaggerated airway narrowing in asthmatics is an abnormality of the properties of airway smooth muscle (Martin et al., 2000; Seow et al., 1998). Rapid relief from airway limitation in asthmatic patients by b-stimulant inhalation may also suggest an involvement

Correspondence to: Yoshihiko Chiba, Ph.D., Department of Pharmacology, School of Pharmacy, Hoshi

University, 2-4-41 Ebara, Shinagawa-ku, Tokyo 142-8501, Japan

Tel: 81-3-5498-5786 Fax: 81-3-5498-5787 e-mail: chiba@hoshi.ac.jp 
of augmented airway smooth muscle contraction in the airway obstruction. Thus, it may be important for development of asthma therapy to understand changes in the contractile signaling of airway smooth muscle cells associated with the disease.

There is increasing evidence that a monomeric GTP-binding protein, RhoA, and its downstream target, Rho-kinases, are involved in the $\mathrm{Ca}^{2+}$-independent contraction (termed $\mathrm{Ca}^{2+}$ sensitization) of airway smooth muscles (Chiba et al., 1999; 2005; Ito et al., 2001; Yoshii et al., 1999). When the RhoA/Rho-kinase system is activated by contractile agonists, the activity of myosin light chain (MLC) phosphatase is reduced and the level of phosphorylated MLC is in turn increased, resulting in an augmentation of contraction. Recent studies demonstrated that the agonist-induced, RhoA/Rho-kinase-mediated $\mathrm{Ca}^{2+}$ sensitization of bronchial smooth muscle (BSM) contraction is augmented in rats (Chiba et al., 1999) and mice (Chiba et al., 2005) with allergic bronchial asthma. An importance of the RhoA/Rho-kinase system has also been demonstrated in human BSM (Yoshii et al., 1999), and the signaling of RhoA and its downstream Rho-kinases are now considered as a therapeutic target for the treatment of airway hyperresponsiveness in asthma (Gosens et al., 2006; Kume, 2008; Schaafsma et al., 2008a; 2008b).

Interleukin-4 (IL-4), one of the T-helper 2 (Th2) cytokines, is believed to play a role in asthma (Dabbagh et al., 1999; Steinke and Borish, 2001; Batra et al., 2004; Bryborn et al., 2004). An increased expression of IL-4 has been demonstrated in bronchoalveolar lavage fluid after segmental allergen challenge to asthmatic patients (Batra et al., 2004). IL-4 promotes IgE production, and eosinophilic airway inflammation by increasing eotaxin expression and inhibiting eosinophil apoptosis (Steinke and Borish, 2001). IL-4 induces mucus hypersecretion (Dabbagh $e t$ $a l ., 1999)$ that contributes to airway obstruction. Interestingly, IL-4 also acts on airway smooth muscle directly, and has an ability to cause hyperresponsiveness of airway smooth muscle (Bryborn et al., 2004). IL-4 is known to share many functional properties with IL-13 (Hershey, 2003). Recent studies revealed that IL-13 caused an increase in RhoA mRNA via an activation of signal transducer and activator of transcription factor 6 (STAT6) in human bronchial smooth muscle cells (Chiba et al., 2009a; 2009b; 2009c). This reminds us of an idea that IL-4 also has an ability to increase RhoA transcription. To confirm the hypothesis, the effects of IL-4 on RhoA mRNA expression and promoter activity of RhoA gene were examined in cultured human BSM cells (hBSMCs).

\section{Methods}

\section{Cell culture and RNA extraction}

Normal human bronchial smooth muscle cells (hBSMCs; Cambrex Bio Science Walkersville, Inc., Walkersville, MD, USA) were maintained in SmBM medium (Cambrex) supplemented with $5 \%$ fetal bovine serum, $0.5 \mathrm{ng} / \mathrm{mL}$ human epidermal growth factor (hEGF), $5 \mu \mathrm{g} / \mathrm{mL}$ insulin, $2 \mathrm{ng} /$ $\mathrm{mL}$ human fibroblast growth factor-basic (hFGF-b), $50 \mu \mathrm{g} / \mathrm{mL}$ gentamicin and $50 \mathrm{ng} / \mathrm{mL}$

amphotericin B. Cells were maintained at $37^{\circ} \mathrm{C}$ in a humidified atmosphere $\left(5 \% \mathrm{CO}_{2}\right)$, fed every 48-72 hr, and passaged when cells reached $90-95 \%$ confluence. Then the hBSMCs (passages 57) were seeded in 6-well plates (Becton Dickinson Labware, Franklin Lakes, NJ, USA) at a density of 3,500 cells $/ \mathrm{cm}^{2}$ and, when $80-85 \%$ confluence was observed, cells were cultured without serum 
for $24 \mathrm{hr}$ before addition of recombinant human IL-4 (10-100 ng/mL; PeproTech EC, Ltd., London, UK). Twenty-four hr after the IL-4 treatment, cells were washed with PBS, immediately collected and disrupted with $1 \mathrm{~mL} /$ well of TRI Reagent (Sigma-Aldrich, St. Louis, MO, USA). Total RNAs were extracted according to the manufacturer's instructions.

\section{Real-time RT-PCR for RhoA $m R N A$}

The mRNA levels of RhoA were examined by real-time RT-PCR as described previously (Chiba et al., 2009b). Briefly, cDNAs were prepared from the total RNA (1 mg) using QuantiTectTM reverse transcription kit (Qiagen Inc., Valencia, CA, USA) according to the manufacturer's instructions. Then the RT reaction mixture $(1 \mu \mathrm{L})$ was subjected to real-time PCR analyses using iQTM SYBR Green Supermix (Bio-Rad Laboratories, Hercules, CA, USA) according to the manufacturer's instructions. The thermal cycle profile used was 1 ) denaturing for $15 \mathrm{sec}$ at $95^{\circ} \mathrm{C}, 2$ ) annealing primers for $30 \mathrm{sec}$ at $55^{\circ} \mathrm{C}$, and 3) extending the primers for $30 \mathrm{sec}$ at $72^{\circ} \mathrm{C}$. The PCR amplification was performed at 40 cycles with monitoring fluorescence. The primer sets used were: QuantiTect Primer Assay, QT00044723 for human RhoA and QT00079247 for human GAPDH (Qiagen Inc.).

\section{Construction of human RhoA-luciferase promoter plasmids}

For the reporter assay, a human RhoA genomic fragment from -1248 bp to +21 bp (the transcription initiation site is +1 ) was obtained by PCR amplification using the human genomic DNA isolated from hBSMCs as a template. The forward primer contained a $K p n I$ restriction site and reverse primer contained a $B g l \mathrm{II}$ restriction site. The PCR primer sets used were as follows: 5'-GACTCCGGGAGCTCAAAATAGC-3' (sense) and 5'-GCGCACTCACAGATCTTCCACTAT-3' (antisense), which were designed from published sequences (GenBank Accesion No. NC_000003). The PCR product was digested by $K p n \mathrm{I}(-1241)$ and $B g l \mathrm{II}(+8)$, and inserted into the pGL4.10 vector (Promega, Madison, WI, USA). Utilizing the resultant plasmid vector as a template, four 5'deletion constructs (pGL4-564, -342, -197 and -112, that contains four, three, two and one STATs binding region, respectively; see Fig. 2) were also prepared, and sequenced to determine the exact sequences.

\section{Luciferase assay}

The hBSMCs were seeded in 96-well tissue culture plates, grown to $80 \%$ confluence, and transfected with pGL4 reporter plasmids containing various lengths of the human RhoA gene promoter using Lipofectamine 2000 (Invitrogen Life Technologies, Carlsbad, CA, USA) according to manufacturer's instructions. Cells were cultured without serum for $24 \mathrm{~h}$ before addition of recombinant human IL-4. Luciferase assay was performed using ONE-GloTM Luciferase assay system (Promega) $72 \mathrm{~h}$ after transfection in accordance with the manufacturer's instructions. Luciferase activity was measured with a Wallac 1420 ARVOsx multilabel counter (PerkinElmer, Waltham, MA, USA).

\section{Statistical analyses}

In the real-time PCR analyses, the comparative threshold cycle $\left(\mathrm{C}_{\mathrm{T}}\right)$ method was used for 


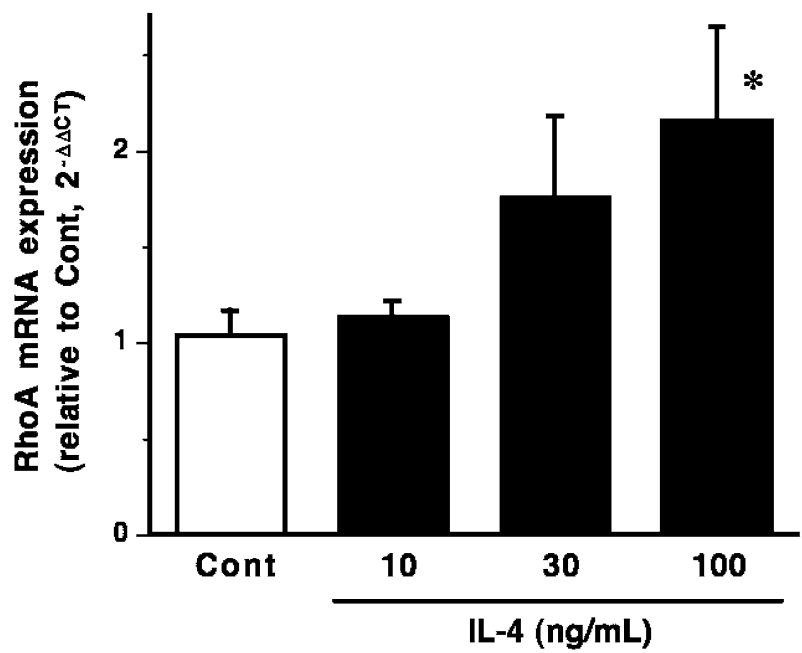

Fig. 1. Effects of IL-4 on the expression of RhoA mRNA in cultured human bronchial smooth muscle cells. After the 24-hr starvation period, cells were incubated with IL-4 (10-100 $\mathrm{ng} / \mathrm{mL}$ ) for $24 \mathrm{hr}$. The gene expression was determined by quantitative real-time RTPCR as described in METHODS section. Each column represents the mean with SEM from 5 independent experiments. *, $P<0.05$ control (Cont; no IL-4 treatment) by Bonferroni/Dunn test.

relative quantification of the target genes (Chiba et al., 2009b). Differences in the $\mathrm{C}_{\mathrm{T}}$ values $\left(\Delta \mathrm{C}_{\mathrm{T}}\right)$ between RhoA and GAPDH were calculated to determine the relative expression levels, using the following formula: $\Delta \mathrm{DC}_{\mathrm{T}}=\left(\Delta \mathrm{C}_{\mathrm{T}}\right.$ of the treated sample $)-\left(\Delta \mathrm{C}_{\mathrm{T}}\right.$ of the control sample $)$. The relative expression level between the samples was calculated according to the equation $2^{-\Delta \Delta C T}$.

All the data are expressed as the mean with S.E. Statistical significance of difference was determined by analysis of variance (ANOVA) with post hoc Bonferroni/Dunn (StatView for Macintosh ver. 5.0, SAS Institute, Inc., NC). A value of $P<0.05$ was considered significant.

\section{Results}

Figure 1 shows the effect of incubation with IL-4 on the expression level of RhoA mRNA in cultured human bronchial smooth muscle cells (hBSMCs). The quantitative real-time RT-PCR revealed that IL-4 caused an increase in RhoA mRNA in a concentration-dependent manner: a significant increase was observed when hBSMCs were incubated with $100 \mathrm{ng} / \mathrm{mL}$ of IL-4 for $24 \mathrm{hr}$ $(P<0.05$; Fig. 1).

The analysis of the 5'-flanking region of the human RhoA gene using the TFSEARCH program (http://mbs.cbrc.jp/research/db/TFSEARCH.html) revealed that it contains four STATs binding regions, e.g., -78 to -70 (score 85.6 ), -191 to -183 (score 80.8), -271 to -263 (score 86.5 ) and -518 to -510 (score 84.6 ) (see Fig. 2). So in the present study, the reporter assay was performed using hBSMCs transfected with plasmid containing $-564 /+8$ bp upstream of the human RhoA gene (named D0 construct) in the absence or presence of IL-4 $(100 \mathrm{ng} / \mathrm{mL})$. As shown in Fig. 2, the promoter activity was markedly and significantly increased by the IL-4 stimulation. The deletions 


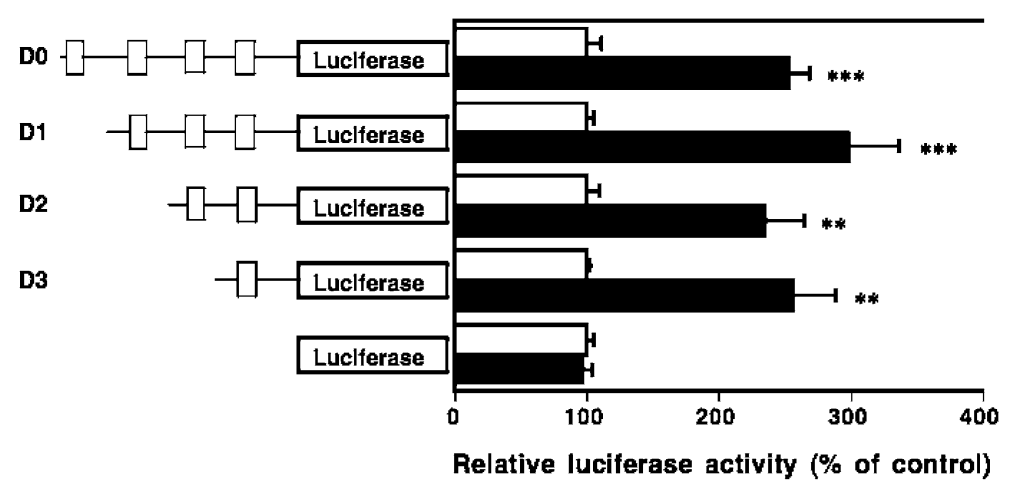

Fig. 2. Determination of the IL-4-responsive region in the human RhoA gene promoter. Cultured human bronchial smooth muscle cells were transfected with luciferase reporter plasmids containing various lengths (D0: -564 , D1: -342 , D2: -197 and D3: $-112 /+8$ bp) of the human RhoA gene promoter. Putative STATs binding regions ( -518 to -510 , 271 to $-263,-191$ to -183 , and -78 to $-70 \mathrm{bp}$ ) are indicated as boxes on the left illustrations. Luciferase assays were performed with (closed columns) or without (open columns; control) stimulation with $100 \mathrm{ng} / \mathrm{mL}$ of IL-4. Each column represents the mean with SEM from 5 independent experiments. **, $P<0.01$ and $* * *, P<0.001$ vs. respective control by Bonferroni/Dunn test.

of $-564 /-343$ bp (D1, which contains three STATs binding resions), $-564 /-198$ bp (D2, which contains two STATs binding resions), and $-564 /-113$ bp (D3, which contains only the most proximal STATs binding region) had no effect on the increased luciferase activity (Fig. 2). These findings indicate that at least the most proximal STATs binding region is required for the IL-4induced increase in promoter activity of the human RhoA gene.

\section{Discussion}

Our previous studies revealed that, in addition to IL-13 (Chiba et al., 2009a; 2009b; 2009c), IL4 is capable of inducing RhoA protein upregulation in hBSMCs (Chiba et al., 2010). The current study suggests that an increased RhoA mRNA expression is, at least in part, one of the causes of RhoA protein upregulation induced by IL-4 (Fig. 1). The reporter gene analyses indicate that IL4 is capable of promoting RhoA transcription, and that the most proximal STATs binding region (78-70 bp upstream of the transcription initiation site) is indispensable to the induction of RhoA transcription by IL-4 stimulation (Fig. 2).

Airway smooth muscle is an important effector tissue regulating bronchomotor tone. It has been suggested that modulation of airway smooth muscle by inflammatory mediators such as cytokines may play a pivotal role in the development of airway hyperresponsiveness (Amrani and Panettieri, 2002), one of the characteristic features of patients with allergic bronchial asthma. In animal models of allergic bronchial asthma, an in vivo airway hyperresponsiveness accompanied by the increased IgE production and pulmonary eosinophilia has been demonstrated (Misawa and Chiba, 1993; Kato et al., 1999; Chiba et al., 2008). In these models of airway hyperresponsiveness, an increased contractility of isolated BSM to contractile agonists has also been found (Misawa and 
Chiba, 1993; Chiba and Misawa, 1995; Chiba et al., 2005; 2008; 2009a). The augmented BSM contraction induced by antigen exposure has reportedly been associated with an upregulation of RhoA (Chiba et al., 1999; 2003; 2005; 2008; 2009a; 2009b; 2009c), a small GTPase that is involved in the agonist-induced $\mathrm{Ca}^{2+}$ sensitization of smooth muscle contraction (Somlyo and Somlyo 2003; Chiba and Misawa, 2004). The RhoA and its downstream Rho-kinases are now considered as a target of airway obstructive diseases such as asthma (Gosens et al., 2006; Kume, 2008; Schaafsma et al., 2008a; 2008b).

Although the promoter of the human RhoA gene is not fully understood until now to our knowledge, IL-4 is known to share many functional properties with IL-13 (Hershey, 2003). We have recently reported that both the increased BSM contractility and the upregulation of RhoA protein observed in the mouse model of allergic asthma were reproduced by IL-13 (Chiba et al., 2009a). The IL-13-induced upregulation of RhoA protein was inhibited by a STAT6 inhibitor AS1517499 or a small interfering RNA (siRNA) for STAT6 (Chiba et al., 2009c), indicating that an activation of STAT6 is required for the RhoA upregulation induced by IL-13. In the present study, the reporter gene analyses revealed that the IL-4-induced increase in promoter activity of the human RhoA gene was disappeared by the depletion of the most proximal STATs binding region (Fig. 2). The importance of this region was also demonstrated by mutation analyses in the IL-13stimulated hBSMCs (Goto et al., 2010). In addition, recent our study also revealed that the RhoA protein upregulation induced by IL-4 was inhibited by co-incubation with AS1517499 in hBSMCs (Chiba et al., 2010). It is thus possible that an activation of STAT6 is also involved in the increased promoter activity of the human RhoA gene induced by IL-4 stimulation.

In conclusion, the present study revealed that IL-4 is capable of upregulating RhoA by promoting its transcription in hBSMCs. The proximal STATs binding region is required for the IL-4-induced increase in promoter activity of the human RhoA gene.

\section{Acknowledgments}

This work was partly supported by the Ministry of Education, Culture, Sports and Technology of Japan.

\section{References}

Amrani, Y. and Panettieri, R.A. Jr. (2002). Modulation of calcium homeostasis as a mechanism for altering smooth muscle responsiveness in asthma. Curr. Opin. Allergy Clin. Immunol. 2: 39-45.

Batra, V., Musani, A.I., Hastie, A.T., Khurana, S., Carpenter, K.A., Zangrilli, J.G. and Peters, S.P. (2004). Bronchoalveolar lavage fluid concentrations of transforming growth factor (TGF)-beta1, TGFbeta2, interleukin (IL)-4 and IL-13 after segmental allergen challenge and their effects on alphasmooth muscle actin and collagen III synthesis by primary human lung fibroblasts. Clin. Exp. Allergy 34: 437-444.

Bryborn, M., Adner, M. and Cardell, L.O. (2004). Interleukin-4 increases murine airway response to kinins, via up-regulation of bradykinin B1-receptors and altered signalling along mitogen-activated protein kinase pathways. Clin. Exp. Allergy 34: 1291-1298.

Chiba, Y., Arima, J., Sakai, H. and Misawa, M. (2008). Lovastatin inhibits bronchial hyperresponsiveness by 
reducing RhoA signaling in rat allergic asthma. Am. J. Physiol. 294: L705-L713.

Chiba, Y. and Misawa, M. (1995). Alteration in $\mathrm{Ca}^{2+}$ availability involved in antigen-induced airway hyperresponsiveness in rats. Eur. J. Pharmacol. 278: 79-82.

Chiba, Y. and Misawa, M. (2004). The role of RhoA-mediated $\mathrm{Ca}^{2+}$ sensitization of bronchial smooth muscle contraction in airway hyperresponsiveness. J. Smooth Muscle Res. 40: 155-167.

Chiba, Y., Nakazawa, S., Todoroki, M., Shinozaki, K., Sakai, H. and Misawa, M. (2009a). Interleukin-13 augments bronchial smooth muscle contractility with an up-regulation of RhoA protein. Am. J. Respir. Cell Mol. Biol. 40: 159-167.

Chiba, Y., Sakai, H., Wachi, H., Sugitani, H., Seyama, Y. and Misawa, M. (2003). Upregulation of rhoA mRNA in bronchial smooth muscle of antigen-induced airway hyperresponsive rats. J. Smooth Muscle Res. 39: 221-228.

Chiba, Y., Takada, Y., Miyamoto, S., Mitsui-Saito, M., Karaki, H. and Misawa, M. (1999). Augmented acetylcholine-induced, Rho-mediated $\mathrm{Ca}^{2+}$ sensitization of bronchial smooth muscle contraction in antigen-induced airway hyperresponsive rats. Br. J. Pharmacol. 127: 597-600.

Chiba, Y., Tanabe, M., Goto, K., Sakai, H. and Misawa, M. (2009b). Down-regulation of miR-133a contributes to up-regulation of Rhoa in bronchial smooth muscle cells. Am. J. Respir. Crit. Care Med. 180: 713-719.

Chiba, Y., Todoroki, M. and Misawa M. (2010). Interleukin-4 upregulates RhoA protein via an activation of STAT6 in cultured human bronchial smooth muscle cells. Pharmacol. Res. 61: 188-192.

Chiba, Y., Todoroki, M., Nishida, Y., Tanabe, M. and Misawa M. (2009c). A novel STAT6 inhibitor AS1517499 ameliorates antigen-induced bronchial hypercontractility in mice. Am. J. Respir. Cell Mol. Biol. 41: 516-524.

Chiba, Y., Ueno, A., Shinozaki, K., Takeyama, H., Nakazawa, S., Sakai, H. and Misawa, M. (2005). Involvement of RhoA-mediated $\mathrm{Ca}^{2+}$ sensitization in antigen-induced bronchial smooth muscle hyperresponsiveness in mice. Respir. Res. 6: Art. No. 4.

Dabbagh, K., Takeyama, K., Lee, H.M., Ueki, I.F., Lausier, J.A. and Nadel, J.A. (1999). IL-4 induces mucin gene expression and goblet cell metaplasia in vitro and in vivo. J. Immunol. 162: 6233-6237.

Eder, W., Ege, M.J. and von Mutius, E. (2006). The asthma epidemic. N. Engl. J. Med. 355: 2226-2235.

Gosens, R., Schaafsma, D., Nelemans, S.A. and Halayko, A.J. (2006). Rho-kinase as a drug target for the treatment of airway hyperresponsiveness in asthma. Mini Rev. Med. Chem. 6: 339-348.

Goto, K., Chiba, Y., Matsusue, K., Hattori, Y., Maitani, Y., Sakai, H., Kimura, S. and Misawa, M. (2010). The proximal STAT6 and NF-kB sites are responsible for RhoA transcription in human bronchial smooth muscle. Pharmacol. Res. 61: 466-472.

Hershey, G.K. (2003). IL-13 receptors and signaling pathways: an evolving web. J. Allergy Clin. Immunol. 111: $677-690$.

Ito, S., Kume, H., Honjo, H., Katoh, H., Kodama, I., Yamaki, K. and Hayashi, H. (2001). Possible involvement of Rho kinase in $\mathrm{Ca}^{2+}$ sensitization and mobilization by $\mathrm{MCh}$ in tracheal smooth muscle. Am. J. Physiol. 280: L1218-L1224.

Kato, Y., Manabe, T., Tanaka, Y. and Mochizuki, H. (1999). Effect of an orally active Th1/Th2 balance modulator, M50367, on IgE production, eosinophilia, and airway hyperresponsiveness in mice. $J$. Immunol. 162: 7470-7479.

Kume, H. (2008). RhoA/Rho-kinase as a therapeutic target in asthma. Curr. Med. Chem. 15: 2876-2885.

Martin, J.G., Duguet, A. and Eidelman, D.H. (2000). The contribution of airway smooth muscle to airway narrowing and airway hyperresponsiveness in disease. Eur. Respir. J. 16: 349-354.

Misawa, M. and Chiba, Y. (1993). Repeated antigenic challenge-induced airway hyperresponsiveness and airway inflammation in actively sensitized rats. Jpn. J. Pharmacol. 61: 41-50.

Schaafsma, D., Gosens, R., Zaagsma, J., Halayko, A.J. and Meurs, H. (2008a). Rho kinase inhibitors: a novel therapeutical intervention in asthma? Eur. J. Pharmacol. 585: 398-406.

Schaafsma, D., Roscioni, S.S., Meurs, H. and Schmidt, M. (2008b). Monomeric G-proteins as signal 
transducers in airway physiology and pathophysiology. Cell. Signal. 20: 1705-1714.

Seow, C.Y., Schellenberg, R.R. and Pare, P.D. (1998). Structural and functional changes in the airway smooth muscle of asthmatic subjects. Am. J. Respir. Crit. Care Med. 158: S179-S186.

Somlyo, A.P. and Somlyo, A.V. (2003). $\mathrm{Ca}^{2+}$ sensitivity of smooth muscle and nonmuscle myosin II: modulated by G proteins, kinases, and myosin phosphatase. Physiol. Rev. 83: 1325-1358.

Steinke, J.W. and Borish, L. (2001). Th2 cytokines and asthma. Interleukin-4: its role in the pathogenesis of asthma, and targeting it for asthma treatment with interleukin-4 receptor antagonists. Respir. Res. 2: $66-70$.

Yoshii, A., Iizuka, K., Dobashi, K., Horie, T., Harada, T., Nakazawa, T. and Mori, M. (1999). Relaxation of contracted rabbit tracheal and human bronchial smooth muscle by Y-27632 through inhibition of $\mathrm{Ca}^{2+}$ sensitization. Am. J. Respir. Cell Mol. Biol. 20: 1190-1200. 